\title{
Genetic features and application value of next generation sequencing in the diagnosis of synchronous multifocal lung adenocarcinoma
}

\author{
XIAOYAN CHEN ${ }^{1-3^{*}}$, JUN LU $^{1 *}$, YINGYING WU $^{1}$, XINGRAN JIANG $^{1}$, \\ YAJUAN GU ${ }^{1}$, YUNLONG LI ${ }^{1}$, HONGYING ZHAO ${ }^{1}$ and MULAN JIN ${ }^{1}$ \\ ${ }^{1}$ Department of Pathology, Capital Medical University, Affiliated Beijing Chaoyang Hospital, Beijing 100020; \\ ${ }^{2}$ Department of Pathology, Baotou Medical College of Inner Mongolia University of Science and Technology; \\ ${ }^{3}$ Department of Pathology, The First Affiliated Hospital of Baotou Medical College, Inner Mongolia \\ University of Science and Technology, Baotou, Inner Mongolia 014010, P.R. China
}

Received October 30, 2019; Accepted May 14, 2020

DOI: $10.3892 / \mathrm{ol} .2020 .11843$

\begin{abstract}
The present study aimed to elucidate the genetic features of multiple lung cancer (MLC) and identify effective molecular markers for diagnosis using next generation sequencing (NGS). The present data may also inform patient treatment and prognosis. A total of 35 lesions were obtained from 17 patients with MLC. Based on lesion histology and NGS, 13 cases of multiple primary lung cancer (MPLC) were identified and 4 cases were classified as intrapulmonary metastasis (IPM). All 4 patients with IPM exhibited an epidermal growth factor receptor (EGFR) mutation and synchronous mutation of at least one tumor suppressor gene. The frequency and percentage of EGFR mutations, accompanied with tumor suppressor genes, were significantly higher in patients with IPM compared with MPLC. Furthermore, a high EGFR-heterogeneity score and male sex were risk factors of IPM occurrence. There were significant differences in mean EGFR mutation abundance alone, mutations of tumor suppressor genes and mutations of EGFR combined with tumor suppressor genes between patients with adenocarcinoma (ADC) and adenocarcinoma in situ (AIS). In conclusion, histological characteristics combined with genetic alterations may be an effective method for the diagnosis of MPLC and IPM, and NGS may serve as a useful diagnostic tool. MLC exhibited unique molecular characteristics, including higher
\end{abstract}

Correspondence to: Professor Mulan Jin, Department of Pathology, Capital Medical University, Affiliated Beijing Chaoyang Hospital, 8 Worker's Stadium South Road, Beijing 100020, P.R. China

E-mail: 625966785@qq.com

*Contributed equally

Key words: multiple primary lung cancer, intrapulmonary metastasis, next generation sequencing, mutation, heterogeneity score, tumor cellularity, progression-free survival rates of EGFR mutations, EGFR driver mutations accompanied with tumor suppressor gene mutations and the absence of anaplastic lymphoma kinase mutations, which may help distinguish between patients with MPLC or IPM. The present study hypothesized that the mean frequency of EGFR mutations, mutations of tumor suppressor genes and mutations of both EGFR and tumor suppressor genes may serve an important role in the development of AIS to ADC. The results of the present study highlight the potential underlying mechanisms of lung ADC development, which may assist with future elucidation of effective treatments to prevent the progression of lung cancer.

\section{Introduction}

Identification of two or more primary lung carcinomas at presentation is not uncommon, with an incidence ranging from 5.7-11.5\% between 1985-2002 (1-5). Specifically, a diagnosis of multiple primary lung adenocarcinomas was observed in up to $8 \%$ of 369 patients who underwent pulmonary resection for adenocarcinoma between 1994-2002 $(1,2)$. However, as imaging techniques have improved, the incidence of multiple lung cancer (MLC) has increased. Distinguishing multiple primary lung cancer (MPLC) from intrapulmonary metastasis (IPM) may assist in predicting the outcome and appropriate treatment of patients with this disease (6). However, classifying multiple lung nodules as MPLC or IPM remains challenging using histological typing alone.

Previously, novel diagnostic criteria have been developed to determine whether intrapulmonary polynodules are primary tumors or IPM (7). According to the most recent 8th edition American Joint Committee on Cancer staging manual (AJCC staging manual) (8); i) two or more distinct and histologically different masses were considered MPLC; ii) multiple ground-glass or part-solid nodules, histologically of with lepidic growth pattern were considered MPLC and iii) multiple tumor nodules with the same histological type and/or with same molecular profile were considered IPM $(6,7)$. Genomic alterations were commonly assessed using fluorescence in-situ 
hybridization and NGS $(6,9)$. These criteria have emphasized the need for a combination of diagnostic approaches, including clinical, histopathological and molecular diagnoses. However, to the best of our knowledge, there is still no universally accepted standard diagnostic method for patients with MLC. Previously, studies have reported that the use of next generation sequencing (NGS) may be a promising tool for patient diagnosis based on the hypothesis that clonally related (IPM) and independent tumors (MPLC) exert different patterns of mutational concordance (10-12). In theory, the simultaneous identification of multiple tumor driver genes should enable improved distinction between MPLC and IPM $(13,14)$. Xiao et al $(14)$, utilized NGS to investigate non-small cell lung cancer as this cancer type often demonstrates genetic heterogeneity. In their cohort, 1 of the 6 patients with similar comprehensive histological assessment results and EGFR mutation type was identified as having different gene mutation types via NGS, revealing that the patient had synchronous multiple primary lung adenocarcinomas (MPLA). In addition, in a study by Li et al (12), 20 paired tumors (Each patient had two tumors considered as a pair) obtained from 20 patients with synchronous multifocal lung adenocarcinomas were analyzed using NGS. The results revealed no discordance of mutational status in all tumor pairs diagnosed as intrapulmonary metastasis via histological examination, whereas the discordance rate was as high as $61.5 \%$ (8 out of 13) in tumor pairs diagnosed as equivocal or multiple primary cancer tumors. Li et al (12), hypothesized that the mutational status of all multifocal tumors may aid the diagnosis and selection of the most effective treatment strategies. Donfrancesco et al (9), demonstrated that pathological criteria were less accurate compared with molecular criteria when staging MLC. However, pathological criteria can be used in conjunction with molecular analysis, although this method is not ideal as NGS is not available everywhere (9). In addition, a diagnostic lineage test based on genomic rearrangements from mate-pair sequencing has been previously applied for distinguishing independent primary from metastatic lung cancer $(14,15)$. Therefore, the screening and function of these diagnostic markers requires further study. The aim of the present study was to probe and analyze genetic differences between patients with MPLC and IPM using NGS. The results may help identify novel biomarkers for the classification of MLC.

\section{Materials and methods}

Patients. A total of 17 patients were diagnosed with MLC through surgical resection at the Beijing Chao Yang Hospital affiliated to Capital Medical University (Beijing, China) between January 2017 and December 2018. The median age of the patients was 58 years (age range, 45-81 years), 4 men and 13 women were included. All these patients were diagnosed as primary lung carcinoma by pathologists with complete clinical data, including preoperative examination, postoperative treatment and follow-up data. Recurrent cases of lung cancer, incomplete clinical data and lack of postoperative treatment and follow-up records were excluded. Two experienced pathologists reviewed all cases independently, blindly and simultaneously. Lobectomy and wedge resections were performed for seven patients, wedge resections for six patients and lobectomy resections for four patients. Of the MLC cases, 7 were adenocarcinoma (ADC) and adenocarcinoma in situ (AIS), 2 were ADC and squamous cell carcinoma (SCC), 6 were ADC and ADC, and 2 cases were diagnosed with multiple AIS (Table I). A total of 35 resected pulmonary nodules and 17 matched normal tissue samples were obtained from the 17 patients. A total of 16/17 patients each had 2 nodules, one patient had 3 nodules, one normal lung tissue sample was selected from each patient as the control for NGS. The present study was approved by the Medical Ethics Committee of Beijing Chaoyang Hospital, Capital Medical University (Beijing, China) (approval no. 2018-Scientific-311). A patient who came from Beijing signed the informed written consent as a representative. The remaining 16 patients were from other provinces of China, thus these patients were contacted via telephone to obtain verbal informed consent before participation in the present study.

Targeted DNA sequencing of resected pulmonary nodules. All commercial kits were used according to the manufacturer's protocols. Total DNA was extracted from formalin-fixed paraffin-embedded (FFPE) tissue samples (including 35 cancer tissue samples and 17 matched normal tissue samples), using the QIAamp DNA FFPE Tissue kit (buffer ATL, buffer AL, buffer AW1, buffer AW2, buffer ATE and proteinase K; Qiagen, Inc.). DNA concentration was subsequently measured using a Qubit dsDNA assay (Thermo Fisher Scientific, Inc.). The quality of genomic DNA was assessed and samples with an A260/A280 ratio of 1.8-2.0 were selected for subsequent analysis. DNA was profiled using a Lung Plasma panel (Guangzhou Burning Rock Medical Laboratory Co., Ltd.), which included 168 cancer-associated genes. The concentration of DNA within samples was measured using a Qubit dsDNA assay (Thermo Fisher Scientific, Inc.), the results of which determined that all samples contained >40 ng DNA. Subsequently, 200-400 bp fragments were selected for analysis using the Agencourt AMPure XP kit (Beckman Coulter, Inc.), and the sequencing libraries were prepared using the NEBNext Ultra II DNA Library Prep kit for Illumina (cat. no. E7645), according to the manufacturer's protocols. A bioanalyzer high-sensitivity DNA assay (Qubit 2.0; Thermo Fisher Scientific, Inc.) was performed to assess the quality and size of DNA samples. Available indexed samples were sequenced using a MiSeq system (Illumina, Inc.) with paired end reads.

Sequencing analysis. Sequencing data were mapped to the human genome (hg19) using the BWA aligner version 0.7.10 (http://bio-bwa.sourceforge.net). PCR duplicate reads were removed prior to the detection of base substitution. Local alignment optimization and variant calling were performed using GATK version 3.2-2 (Broad Institute, Inc.). DNA translocation analysis was performed using Tophat 2 (Center for Computational Biology, Johns Hopkins University and the Genome Sciences Department) and Factera version 1.4.3 (https://factera.stanford.edu). Insert size distribution and the library complexity of each sample was determined to assess levels of DNA degradation. Different mutation calling thresholds were applied to samples with differing levels of DNA quality to avoid false-positive mutation calls due to DNA damage. Gene variants were filtered using the VarScan 
Table I. Genetic alterations and predominant patterns of each tumor obtained in the present study.

\begin{tabular}{|c|c|c|c|c|c|}
\hline $\begin{array}{l}\text { Patient } \\
\text { no. }\end{array}$ & $\begin{array}{l}\text { Tumor } \\
\text { location }\end{array}$ & $\begin{array}{l}\text { Histological } \\
\text { type }\end{array}$ & $\begin{array}{l}\text { Predominant } \\
\text { histological pattern }\end{array}$ & Gene alterations & $\begin{array}{c}\text { Final } \\
\text { diagnosis }\end{array}$ \\
\hline \multirow[t]{2}{*}{1} & RLL & AIS & Lepidic growth pattern & NRAS p.Q61L; BRAF p.N581S & MPLC \\
\hline & RLL & $\mathrm{ADC}$ & Acinar & BRAF p.V600E & \\
\hline \multirow[t]{2}{*}{2} & RUL & AIS & Lepidic growth pattern & EGFR p.L858R & MPLC \\
\hline & RML & $\mathrm{ADC}$ & Acinar & EGFR p.L858R; TP53 p.V272L & \\
\hline \multirow[t]{3}{*}{3} & LUL & AIS & Lepidic growth pattern & $\begin{array}{l}\text { EPHA3 p.K889N; MET p.K1132T; TP53 p.V172F; } \\
\text { MET c.2888-24_2888-8del; RB1 p.G449E }\end{array}$ & MPLC \\
\hline & LUL & $\mathrm{ADC}$ & Lepidic $70 \%$, Acinar $30 \%$ & $\begin{array}{l}\text { RB1 p.G449E; MET c.2888-24_2888-8del; } \\
\text { MET p.D1010Y EPHA3 p.K889N; TP53 p.V172F }\end{array}$ & \\
\hline & LUL & $\mathrm{ADC}$ & $\begin{array}{l}\text { Lepidic } 75 \% \text {, Acinar } 10 \% \text {, } \\
\text { Papillary } 15 \%\end{array}$ & APC p.E538K; EGFR p.L861Q & \\
\hline \multirow[t]{2}{*}{4} & LUL & $\mathrm{ADC}$ & Lepidic & EGFR p.E746_A750del & MPLC \\
\hline & LUL & AIS & Lepidic growth pattern & EGFR p.L858R & \\
\hline \multirow[t]{2}{*}{5} & LLL & AIS & Lepidic growth pattern & EGFR p.L858R; FGFR3 cn_del & MPLC \\
\hline & LLL & $\mathrm{ADC}$ & Lepidic & EGFR p.L858R; ERBB2 cn_amp & \\
\hline \multirow[t]{2}{*}{6} & RLL & AIS & Lepidic growth pattern & EGFR p.L858R & MPLC \\
\hline & RUL & $\mathrm{ADC}$ & Acinar & TP53 p.E287; EGFR p.L858R & \\
\hline \multirow[t]{2}{*}{7} & RLL & AIS & Lepidic growth pattern & EGFR p.L858R & MPLC \\
\hline & RUL & $\mathrm{ADC}$ & Acinar & $\begin{array}{l}\text { FGF3 p.N158=; SMARCA4 cn_amp; PIK3CA } \\
\text { p.E545K EGFR p.L747_P753delinsS; MYC p.E432K }\end{array}$ & \\
\hline \multirow[t]{2}{*}{8} & LLL & $\mathrm{ADC}$ & Acinar & $\begin{array}{l}\text { CCNE1 p.V352I; TP53 c.994-1G>A; SMAD4 } \\
\text { p.R380_G386delinsS; EGFR cn_amp; EGFR } \\
\text { p.L858R; PIK3R1 NA; FGFR1 NA }\end{array}$ & IPM \\
\hline & LUL & $\mathrm{ADC}$ & Acinar & $\begin{array}{l}\text { BRINP3 p.S470=; CCNE1 p.V352I; TP53 c.994-1G>A; } \\
\text { SMAD4 p.R380_G386delinsS; EGFR cn_amp; EGFR } \\
\text { p.L858R }\end{array}$ & \\
\hline \multirow[t]{2}{*}{9} & RLL & $\mathrm{ADC}$ & Acinar & $\begin{array}{l}\text { EMSY p.T288R; EGFR p.L858R; TP53 p.S241C; } \\
\text { CDKN2A p.Y44fs }\end{array}$ & IPM \\
\hline & RUL & ADC & Acinar & $\begin{array}{l}\text { EMSY p.T288R; TP53 p.S241C; EGFR p.L858R; } \\
\text { EGFR cn_amp }\end{array}$ & \\
\hline \multirow[t]{2}{*}{10} & RUL & $\mathrm{ADC}$ & $\begin{array}{l}\text { Lepidic } 50 \% \text {, Acinar } 40 \% \text {, } \\
\text { Papillary } 10 \%\end{array}$ & EGFR p.L858R; TP53 p.R249S & IPM \\
\hline & RML & $\mathrm{ADC}$ & Lepidic $70 \%$, Acinar $30 \%$ & EGFR p.L858R; TP53 p.R249S & \\
\hline \multirow[t]{2}{*}{11} & LLL & $\mathrm{ADC}$ & Acinar & $\begin{array}{l}\text { TP53 p.E224D; EGFR p.E746_T751delinsA; } \\
\text { EGFR cn_amp }\end{array}$ & IPM \\
\hline & LUL & $\mathrm{ADC}$ & Lepidic $70 \%$, Acinar $30 \%$ & $\begin{array}{l}\text { TP53 p.E224D; IL7R p.C349*; EGFR } \\
\text { p.E746_T751delinsA; EGFR cn_amp }\end{array}$ & \\
\hline \multirow[t]{2}{*}{12} & RLL & AIS & Lepidic growth pattern & ERBB2 p.Y772_A775dup & MPLC \\
\hline & RUL & AIS & Lepidic growth pattern & KRAS p.G12D & \\
\hline \multirow[t]{2}{*}{13} & RUL & AIS & Lepidic growth pattern & EGFR p.L858R & MPLC \\
\hline & RUL & AIS & Lepidic growth pattern & EGFR p.L858R & \\
\hline \multirow[t]{2}{*}{14} & RLL & $\mathrm{SCC}$ & Middle-low differentiation & $\begin{array}{l}\text { TP53 c.97-11C>G; CHEK1 p.S251L; EPHA5 } \\
\text { p.L53=; FGFR1 p.L614M; APC p.E1544 }\end{array}$ & MPLC \\
\hline & RUL & $\mathrm{ADC}$ & Lepidic $70 \%$, Acinar $30 \%$ & KRAS p.G13D; STK11 p.E130; EPHA3 p.N866K & \\
\hline \multirow[t]{2}{*}{15} & RUL & $\mathrm{ADC}$ & Lepidic & Negative & MPLC \\
\hline & RML & ADC & Lepidic & EGFR p.A767_V769dup & \\
\hline \multirow[t]{2}{*}{16} & RUL & $\mathrm{ADC}$ & Lepidic & EGFR p.L861Q; MSH2 p.Q264E; EGFR p.G719A & MPLC \\
\hline & RLL & $\mathrm{ADC}$ & Acinar & EGFR p.E746_A750del; PIK3CA p.H1047L & \\
\hline \multirow[t]{2}{*}{17} & RUL & $\mathrm{ADC}$ & Acinar & BRAF p.V600E & MPLC \\
\hline & RUL & $\mathrm{SCC}$ & Middle-low differentiation & $\begin{array}{l}\text { RET c.1264-1G>T; FGF19 cn_amp; FGF4 cn_amp; } \\
\text { FGF3 cn_amp; CCND1 cn_amp; PIK3CA cn_amp; } \\
\text { SOX2 cn_amp; FBXW7 p.R393=; TP53 p.R273L; } \\
\text { PMS2 p.M136V; PMS2 p.G132=; CDKN2A p.R22P; } \\
\text { CARD11 p.E1096=; TRIM58 p.P297= }\end{array}$ & \\
\hline
\end{tabular}


(Genome Institute, Washington University, USA) filter pipeline. Gene variants loci with a sequencing depth $<100$ were filtered out. At least two and five supporting reads were required for insertions/deletions in FFPE tissue samples, respectively, while eight supporting reads were required for single number variations (SNVs) in the samples, and selected exons and introns of 168 genes were captured. Single nucleotide variants and indels were annotated using dbNSFP (version 30a; http://varianttools. sourceforge.net/Annotation/dbNSFP), Catalogue of Somatic Mutations (version 69; https://cancer.sanger.ac.uk/cosmic) and dbSNP (snp138) databases (ftp.ncbi.nih.gov/snp). Variants with a global minor allele frequency $>1.0 \%$ included in the 1000Genome Project (Phase 3; 1000genomes.org/data) were considered to be common single nucleotide polymorphisms and were removed.

Integrative Genomics Viewer (Broad Institute, Inc.) (16) was used to visualize variants aligned against the reference genome to confirm the accuracy of variant calls by checking for possible strand biases and sequencing errors. Gene-level copy number variation was assessed using a statistic after normalizing read depth at each region by total read number and region size, and correcting for GC-bias using the LOESS algorithm (https://www.weisang.com/en/documentation/loessandlowessalgorithm).

Tumor cellularity assessment. Tumor cellularity was assessed by two pathologists in patients with lung cancer. NGS testing was not conducted with specimens that contained $<5 \%$ tumor cells and could not be macro-dissected. Therefore, based on the study by Li et al (17), a standard of $10 \%$ tumor cells was set. All samples were categorized into three groups based on the estimated percentage of tumor cells: Group 1, 6-19\% tumor cellularity; group 2, 20-30\% tumor cellularity; and group 3, $>30 \%$ tumor cellularity. There were 11 specimens in group 1 , 4 specimens in group 2 and 20 specimens in group 3 .

The heterogeneity scores (HSs) of EGFR and tumor suppressor protein 53 (TP53) were calculated as described previously $(17,18)$. A HS of $<1$ indicated that mutations were present in a subpopulation of tumor cells; a score of 1 suggested that mutations were present in all tumor cells; and a score of $>1$ indicated that copy-number variations may exist in tumor cells (12).

Identification of tumor suppressor gene mutations. Whether a tumor had a tumor suppressor gene mutation or not was determined by the mutation status of TP53, phosphatidylinositol-3 kinase catalytic subunit $\alpha$ (PIK3CA), retinoblastoma 1 (RB1) or serine/threonine kinase 11 (STK11).

Statistical analysis. Statistical analysis was performed using SPSS version 17.0 (SPSS Inc.). Continuous variable homogeneity was analyzed using a two-way independent sample t-tests for normally distributed data and a non-parametric rank sum test for non-normally distributed data ( $\mathrm{Z}$ test was used for comparing EGFR mutation abundance between ADC group and AIS group, and Mann-Whitney U test was used for comparing EGFR mutation abundance between MPLC and IPM). ANOVA was used to test for differences among $\geq 2$ groups followed by Tukey's post hoc test. A Pearson $\chi^{2}$ test and Fisher's exact test were used to analyze the observed differences in frequencies. Multivariate logistic regression analysis was used to evaluate the relative risk of IPM when compared with MPLC, irrespective of cause, and was expressed as the odds ratio (OR) with $95 \%$ confidence intervals (CIs). Progression-free survival (PFS) time was analyzed using the Kaplan-Meier method and survival curves were compared using a log-rank or Renyi test, as appropriate. $\mathrm{P}<0.05$ was considered to indicate a statistically significant difference.

\section{Results}

Alterations of genes. Alterations of the EGFR gene were identified in 13 of the 17 patients enrolled (76.5\%), presenting in 24 of the lesions obtained. Among the ADC lesions, nine exhibited exon 21 L858R, two presented with exon 21 L861Q and five lesions exhibited an exon 19 deletion missense mutation. Additionally, copy number amplification was identified in five ADC lesions. The results also revealed EGFR gene exon 21 L858R alterations in seven AIS lesions. Anaplastic lymphoma kinase (ALK) fusion was not identified in any sample. Kirsten rat sarcoma (KRAS) mutations were identified in 2 lesions of 2 patients and a neuroblastoma rat sarcoma (NRAS) mutation was detected in another patient. The rate of KRAS detection was $(2 / 17,11.76 \%)$. No positive mutations were detected in normal lung tissue in 17 patients. The detailed genetic changes of each case are presented in Table I.

Patients with ADC and AIS. A total of seven lesions in nine patients with AIS and 17 lesions in 12 patients with ADC were found to exhibit EGFR mutations. The results of the non-parametric rank sum test demonstrated that the mean abundance of EGFR mutations in the ADC group was higher compared with that in the AIS group ( $\mathrm{Z}=-2.845, \mathrm{P}=0.004$; data not shown). Analysis of EGFR mutation location in patients with AIS and ADC (EGFR non-L858R and EGFR L858R) revealed that the rate of EGFR L858R mutations in AIS was significantly higher compared with that in ADC $\left(\chi^{2}=4.941\right.$, $\mathrm{P}=0.026$; data not shown). There were no significant differences in the mutation status of any other core driver gene between patients with AIS and $\operatorname{ADC}\left(\chi^{2}=0.688, \mathrm{P}=0.407\right)$.

The mutation status of certain tumor suppressor genes, including TP53, PIK3CA, RB1 and STK11 were statistically different in the ADC group compared with the AIS group $\left(\chi^{2}=7.506, P=0.006\right.$; data not shown). Of the nine cases of AIS, 7 were ADC and AIS, and 2 cases were diagnosed with multiple AIS (Table I). In patients with AIS, only one tumor exhibited a tumor suppressor gene mutation, while 10 did not. In patients with ADC, 13 tumors exhibited a tumor suppressor gene mutation and nine did not. The positive rate of mutations in the tumor suppressor genes was assessed, demonstrating that there was a significantly higher positive rate of tumor suppressor gene mutations in ADC compared with AIS $\left(\chi^{2}=7.506, P=0.006\right.$; data not shown).

EGFR status and tumor suppressor gene mutation analysis in patients with AIS and ADC revealed that no AIS tumor exhibited simultaneous mutations: Eight exhibited one mutation, while three did not exhibit a mutation. In ADC lesions, 11 tumors exhibited simultaneous mutations, eight tumors exhibited one mutation and three did not present any mutations. 


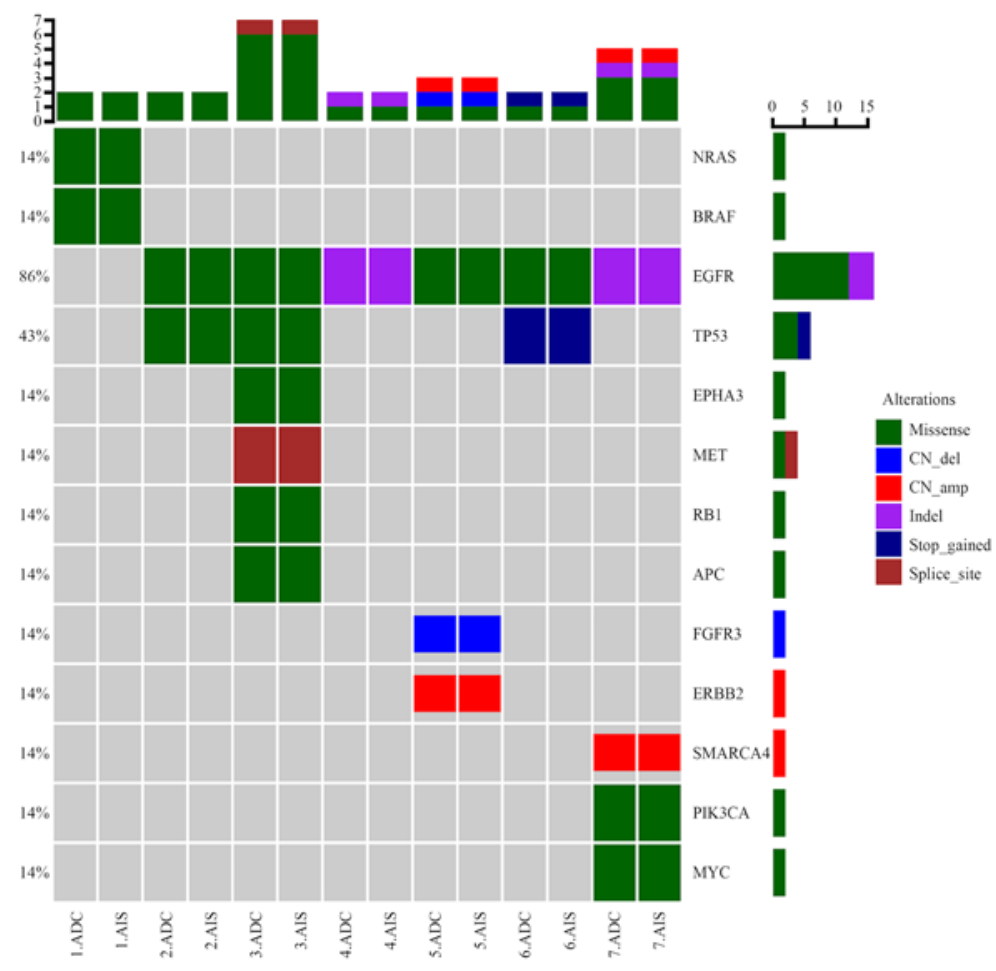

Figure 1. Comparison of genetic changes in 7 patients with simultaneous AIS and ADC. The horizontal axis represents patient number, the left vertical axis represents mutation abundance and the right vertical axis represents gene name. AIS, adenocarcinoma in situ; ADC, adenocarcinoma; CN_del, copy number deletion; CN_amp, copy number amplification.

Following statistical analysis, a significant difference was identified $\left(\chi^{2}=8.250, \mathrm{P}=0.016\right.$; likelihood ratio, $\chi^{2}=11.511$, $\mathrm{P}=0.003$; data not shown), indicating that there were more concurrent EGFR and tumor suppressor gene mutations in ADC lesions compared with AIS lesions.

The results of mean EGFR local mutation abundance analysis revealed that abundance was significantly higher in ADC lesions compared with simultaneous AIS lesions ( $\mathrm{t}=4.598, \mathrm{P}=0.001$; data not shown). However, no significant differences were identified in the positioning of EGFR mutations, the mutation abundance of other core driver genes, mutations in tumor suppressor genes and EGFR mutations accompanied with mutations in tumor suppressor genes (Fig. 1).

MPLC diagnosis. MPLC was diagnosed using histological subtyping and gene alteration analysis. Of the two cases of simultaneous SCC and ADC, it was demonstrated that each exhibited different histological types and different driver gene mutation spectra, which was indicative of multi primary lung cancer. Co-occurrence of AIS and ADC in seven cases and co-occurrence of AIS in 2 cases were diagnosed as MPLC (Table I), according to the AJCC staging manual (7).

Histological subtypes and genetic alteration maps were compared among 6 patients with multiple ADC lesions. Among them, two tumors from patient 16 exhibited different histological subtypes; one tumor exhibited acinar features and one tumor exhibited lepidic features. Both tumors possessed EGFR mutations at different positions (the former: EGFR p.L861Q and EGFR p.G719A, the latter: EGFR p.E746_A750del). In addition, the two tumors from patient 15 exhibited similar histological subtypes (lepidic), but one did not present with any genetic changes, while the other exhibited EGFR driver gene mutations (Table I). As driver gene mutations were different in the 2 aforementioned patients, the diagnosis of MPLC was supported.

The other 4 patients with multiple ADC lesions (patients 8-11) exhibited a similar driver gene mutation spectrum. EGFR and TP53 were detected in the two tumors of patient number 10; however, the abundance of each mutation differed. Among the four patients with multiple ADC lesions, three exhibited similar histological subtypes, (the histological pattern of all four tumors in patient numbers 8 and 9 was acinar, in patient number 10 , the lepidic structure accounted for $50 \%$, acinar $40 \%$ and papillary $10 \%$ in one tumor, the lepidic structure accounted for $70 \%$ and acinar $30 \%$ in another tumor), while one exhibited different histological subtype (in patient number 11, one tumor was dominated by acinar structure, while in another tumor, lepidic structure and acinar structure accounted for 70 and 30\%, respectively; Table I). Combined with the results of NGS, these data supported the diagnosis of IPM (Table I).

The results revealed there was $\sim 8 \%$ (1/13) of discordance of mutational status in all tumor pairs diagnosed as MPLC via histological examination, whereas the discordance rate was $25 \%(1 / 4)$ in tumor pairs diagnosed as intrapulmonary metastasis. However, there was no statistically significant difference $(\mathrm{P}=0.347$; Table SI $)$.

Staging. The stage of all 17 patients was confirmed according to lung cancer staging criteria by AJCC staging manual and NGS sequencing results. The tumor pairs from 13 MPLC 
Table II. Clinicopathological characteristics of patients with MPLC and IPM.

\begin{tabular}{|c|c|c|c|c|}
\hline Clinicopathological characteristic & $\operatorname{MPLC}(n=13)$ & IPM (n=4) & Test value & P-value \\
\hline Age, mean \pm standard deviation $(\min , \max )$ & $61.96 \pm 8.97(47,81)$ & $55.75 \pm 13.89(49,76)$ & $\mathrm{t}=1.16$ & 0.27 \\
\hline Mean tumor size, $\mathrm{cm}(\min , \max )$ & & & $\mathrm{t}=-0.41$ & 0.68 \\
\hline $\mathrm{ADC}$ & $1.79(0.5,4.0)$ & $2.50(1.0,5.0)$ & & \\
\hline AIS & $0.88(0.5,1.7)$ & & & \\
\hline Sex & & & $\chi^{2}=2.04$ & 0.15 \\
\hline Female & 11 & 2 & & \\
\hline Male & 2 & 2 & & \\
\hline Smoker & & & $\chi^{2}=1.12$ & 0.29 \\
\hline Yes & 3 & 0 & & \\
\hline No & 10 & 4 & & \\
\hline Stage & & & $\chi^{2}=5.86$ & 0.02 \\
\hline$<\mathrm{IIB}$ & 9 & 0 & & \\
\hline$\geq \mathrm{IIB}$ & 4 & 4 & & \\
\hline
\end{tabular}

$\mathrm{t}, \mathrm{t}$ test; $\chi^{2}$, Pearson's test. MPLC, multiple primary lung cancer; IPM, intrapulmonary metastasis; ADC, adenocarcinoma; AIS, adenocarcinoma in situ; min, minimum; max, maximum.

patients were staged separately. Different histological types for separate tumor $(\mathrm{T})$, nodal metastases $(\mathrm{N})$ and distant metastases (M) for each tumor, so the two cases of simultaneous SCC and ADC were separate T, N and M for each tumor (data not shown). Co-occurrence of AIS and ADC in seven cases and co-occurrence of AIS in 2 cases were diagnosed as MPLC, the pathological (p)T stage of the largest tumor or main lesion was defined as the highest pT stage (data not shown). IPM usually represented by multiple tumor nodules of the same histological type and/or molecular profile. A separate tumor nodule in the same lobe is staged as T3, in the ipsilateral lobe as T4. A total of four patients with IPM were respectively staged as pathological T4, two tumors of each patient were both on the same side (left or right lung), but not in the same lung lobe, so the four patients with IPM were considered pathological T4 stage (Table II). Because there were only a few cases with TNM stage II B, stage III and IV (data not shown), in order to perform a more reasonable statistical analysis, these were combined into stage $\geq$ IIB, the rest were stage $<$ IIB (including Tis, I and IIA; data not shown).

Comparison of patient characteristics between MPLC and IPM groups. Clinicopathological characteristics of patients with MPLC and IPM are listed in Table II, there were no statistical differences in age, sex, tumor size and smoking status between the two groups (all $\mathrm{P}>0.05$ ), while the tumor TNM stage was significantly higher in the IPM group compared with the MPLC group $(\mathrm{P}<0.05)$. Genetic changes of patients with MPLC and IPM are presented in Fig. 2 and the specific comparisons are listed in Table III. There were 27 tumors in the MPLC group, among which 16 had EGFR mutations. In the present study, significant differences in EGFR mutation abundance were detected when comparing patients with MPLC and IPM, the mean abundance of EGFR mutations was significantly higher in the IPM group compared with the MPLC group $(\mathrm{P}<0.05)$.
Furthermore, the incidences of EGFR driver mutations, involvement of tumor suppressor genes, EGFR accompanied with tumor suppressor gene mutations were significantly higher in the IPM group compared with the MPLC group (all $\mathrm{P}<0.05)$.

Tumor cellularity. EGFR mutation rates were high in each of the three groups (group 1,7/11 64\%; group 2,3/4; 75\%); group 3, $14 / 20,70 \%$ ), despite results not being statistically different ( $P>0.05)$. There was no significant difference between group 1 vs. $2(\mathrm{P}=0.904)$, and group 2 vs. group $3(\mathrm{P}=0.056)$; however, a significant difference was observed between group 1 vs. 3 ( $\mathrm{P}=0.006$; Fig. 3).

HS calculation and comparison. Among the patients with IPM, the mean EGFR HS value was significantly higher compared with patients with MPLC $(\mathrm{t}=3.502$; $\mathrm{P}=0.009$; EGFR $\mathrm{HS}>1$ in IPM), indicating that copy number variations may exist in tumor cells. However, there was no statistical difference in the mean HS value of TP53 between the two groups $(\mathrm{t}=0.151, \mathrm{P}=0.883$; HS $<1$ in both groups; Fig. 4).

Identification of PFS risk factors in patients with MPLC and $I P M$. Univariate regression analysis revealed that EGFR mutation frequency, EGFR HS, TP53 mutation frequency, TP53 HS and sex were all independent risk factors of IPM (Table SII). Furthermore, multivariate dichotomous logistic regression analysis demonstrated that EGFR HS (OR, 760; $\mathrm{P}=0.024$; 95\% CI, 2.443-236,548) and the male sex (OR, 355; $\mathrm{P}=0.048$; 95\% CI, 1.053-120,267) were risk factors of IPM (Table SIII). Therefore, a higher EGFR HS value and the male sex were associated with an increased risk of IPM.

Kaplan-Meier PFS analysis of the 13 patients with MPLC and the 4 patients with IPM are presented in Fig. 5 . The postoperative median PFS time of the 13 patients with MPLC was 29 months and was 16 months for patients with IPM. There 


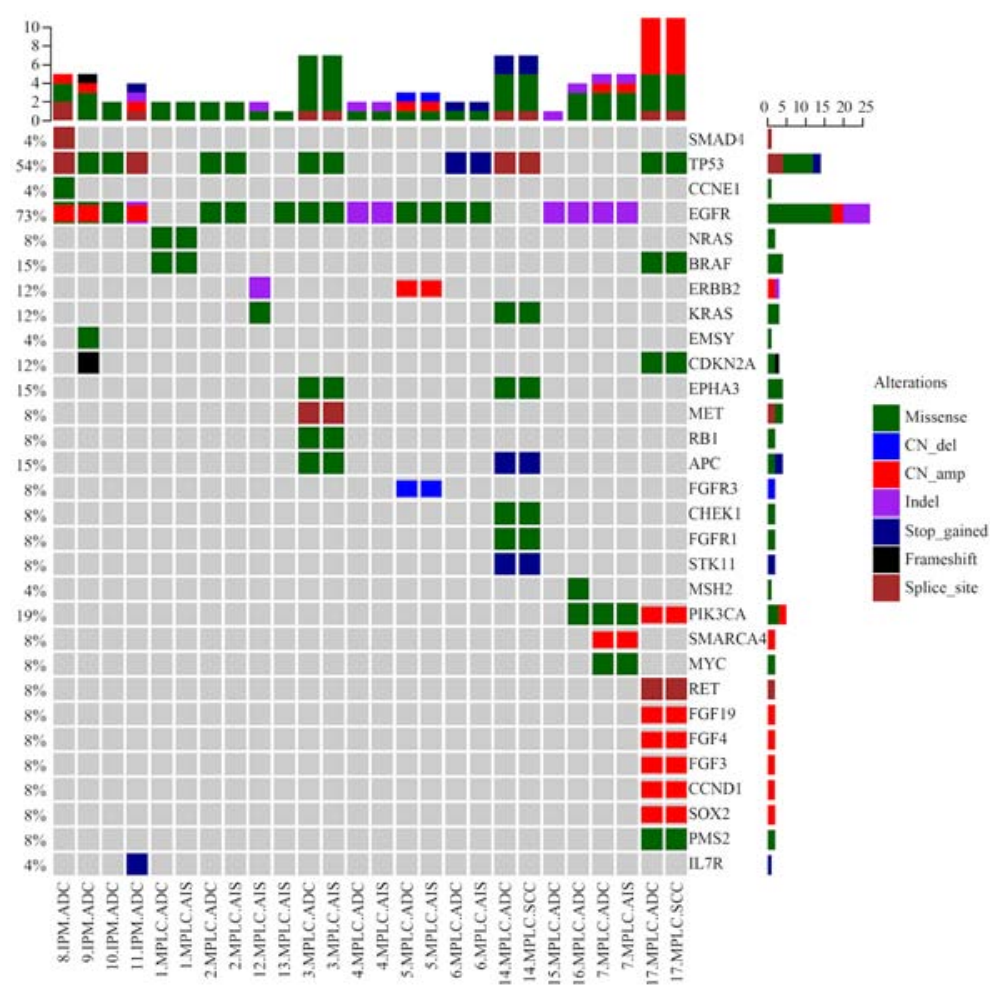

Figure 2. Comparison of genetic changes between patients with MPLC and IPM. The X-axis represents patient number, the left vertical axis represents mutation abundance and the right vertical axis represents gene name. MPLC, multiple primary lung cancer; IPM, intrapulmonary metastasis; CN_del, copy number deletion; CN_amp, copy number amplification.

was no significant difference between the PFS of patients with MPLC and IPM (Renyi P=0.39; log-rank P=0.307).

\section{Discussion}

Comprehensive histological assessment has provided a suitable and reliable method for the identification of MPLCs (19); however, certain patients with $\geq 2$ lung adenocarcinomas exhibit similar pathological subtypes (17). It is therefore difficult to distinguish MLPC from IPM using histology alone. It is now generally accepted that diagnostic criteria should be based on clinical, histopathological and molecular data combined $(6,11)$.

Lung cancer may result from the accumulation of mutations in a branched evolutionary model similar to that of a growing tree (20-24). However, the comprehensive genomic landscape of synchronous multifocal lung adenocarcinomas, primarily those of pre-invasive and invasive lung cancer, have not been extensively compared. Additionally, to the best of our knowledge, correlations between specific clinicopathological characteristics, genes alterations and the prognosis of patients with MPLC or IPM are yet to be performed.

In the present study, four patients were diagnosed with IPM, three of which exhibited similar histological subtypes and genetic changes in each lesion, another one patient exhibited different histological subtypes but similar genetics changes. These patients were diagnosed with IPM for two reasons. Firstly, the two tumors had similar mutations in the main driver gene and thus were classified as IPM, based on the NGS test results and in combination with the literature (6,10-12). Some studies report that the use of NGS appears promising in addressing this challenge in distinguishing MLPC from IPM, based on the hypothesis that clonally related (IPM) and independent tumors (MPLC) exert different patterns of mutational concordance (10-12). Secondly, one patient had adenocarcinoma with two lesions with different histological subtypes. Some tumors had morphological changes after metastasis, and histological subtype changes. Changes in the morphological subtype of lung adenocarcinoma with distant metastases are common in clinical practice. Donfrancesco et al (9) demonstrated that architectural patterns cannot be used to differentiate multiple primary tumors from IPM, the lepidic architecture may be noteworthy to distinguish separate tumor nodules (25) because of the poor repeatability of the results of the lepidic architecture between multiple observers. However, additional studies are required to support this conclusion.

The present results demonstrated that there was $\sim 8 \%$ (1/13) discordance of mutational status in all tumor pairs diagnosed as MPLC via histological examination, whereas the discordance rate was $25 \%$ (1/4) in tumor pairs diagnosed as IPM. However, this difference was not significantly different $(\mathrm{P}=0.347$; Table SI). It has been reported that discrepancy between clinical and molecular classification of originally presumed cases of multiple primary lung cancer tumors range between 18 and $30 \%$ in different study cohorts $(26,27)$. Therefore, histological characteristics used in combination with genetic alteration analysis may be an effective method to diagnose MPLC and IPM. It was hypothesized that the mutational status of all multifocal tumors may aid the diagnosis and selection of the most effective treatment strategies.

In the present study, EGFR gene alterations were identified in $13 / 17$ patients $(76.5 \%)$, which included 24 lesions. 
Table III. Genetic changes of patients with MPLC and IPM.

\begin{tabular}{|c|c|c|c|c|}
\hline Mutation characteristic & $\operatorname{MPLC}(n=27)$ & IPM $(n=8)$ & Test value & P-value \\
\hline EGFR mutation abundance, mean \pm standard deviation & $0.111 \pm 0.075$ & $0.496 \pm 0.278$ & $\mathrm{U}=14$ & 0.002 \\
\hline EGFR mutation site & & & $\chi^{2}=0.375$ & 0.540 \\
\hline L858R & 10 & 6 & & \\
\hline Non-L858R & 6 & 2 & & \\
\hline Driver mutations & & & $\chi^{2}=4.753$ & 0.029 \\
\hline EGFR driver mutations & 16 & 8 & & \\
\hline Non-EGFR driver mutations & 11 & 0 & & \\
\hline Involvement of tumor suppressor genes & & & $\chi^{2}=12.315$ & $<0.01$ \\
\hline Yes & 8 & 8 & & \\
\hline No & 19 & 0 & & \\
\hline EGFR accompanied with tumor suppressor gene mutations & & & $\chi^{2}=22.626$ & $<0.01$ \\
\hline Both mutations simultaneously & 3 & 8 & & \\
\hline One of the mutations & 18 & 0 & & \\
\hline None & 6 & 0 & & \\
\hline
\end{tabular}

U, Mann-Whitney; $\chi^{2}$, Pearson's test. MPLC, multiple primary lung cancer; IPM, intrapulmonary metastasis; EGFR, epidermal growth factor receptor; $n$, number of tumors.

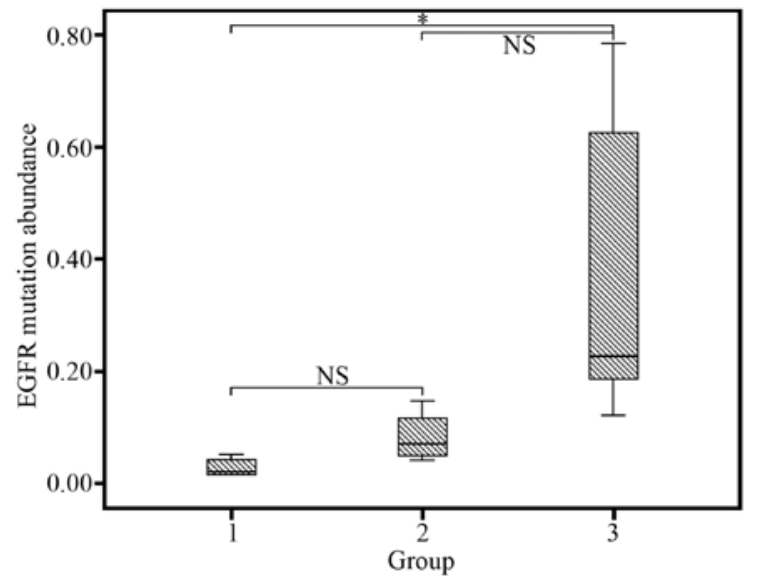

Figure 3. Comparison of EGFR mutation abundance among the three groups Mutation abundance was significantly higher in group 3 when compared with group 1. ${ }^{*} \mathrm{P}<0.05$, group 3 vs. group 1 . There were no statistical differences between group 3 and group 2, also between group 2 and group 1. NS, not significant; EGFR, epidermal growth factor receptor.

Xiao et al (14) observed EGFR mutations in 64 tumors obtained from $35 / 37$ patients $(94.6 \%)$ with synchronous MPLAs. Further studies have revealed higher EGFR mutation frequencies compared with reported rates of single lung adenocarcinoma mutations, ranging between 11 and $51.4 \%$ (28-32). In the present study, significant differences in EGFR mutation abundance were detected when comparing patients with MPLC and IPM (Table III). High rates of EGFR mutations and EGFR abundance may be a result of gene alterations in patients with IPM, therefore aiding the differentiation between MPLC and IPM. However, a larger sample size is required to confirm this.

In the present study, all patients with IPM exhibited EGFR driver mutations, accompanied with a tumor suppressor gene

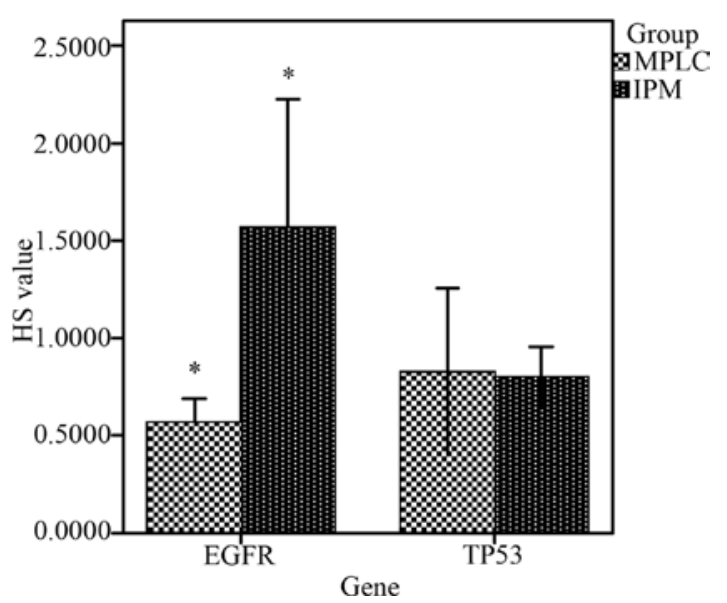

Figure 4. Comparison of EGFR HS and TP53 HS mean values between patients with MPLC and IPM. EGFR HS values were significantly higher in patients with IPM compared with those with MPLC. No significant differences were identified in the mean values of TP53 HS. " $\mathrm{P}<0.05$ vs. IPM. EGFR, epidermal growth factor receptor; HS, heterogeneity score; TP53, tumor protein 53; MPLC, multiple primary lung cancer; IPM, intrapulmonary metastasis.

mutation, which included TP53, PIK3CA, STK11 or RB1 mutations. A recent study that analyzed 17,664 patients with lung cancer identified 2-3 concomitant driver mutations in $\sim 1 \%$ of cases (33). In another large database study, the occurrence of EGFR co-mutations with other cancer driver mutations was $\sim 10 \%$ (34). The results of the present study revealed that the co-mutation rate of EGFR and tumor suppressor genes in the IPM group was markedly higher compared with those presented in other studies with single ADC $(29,33)$. These results may partly explain the mechanism of intrapulmonary metastasis, in which tumor suppressor gene mutations may exert a promotive effect. Furthermore, co-mutations may potentially impair the efficacy of tyrosine kinase inhibitors 


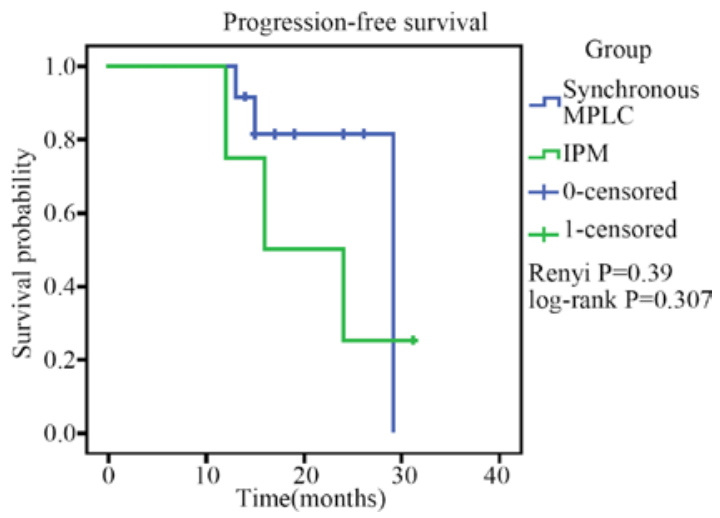

Figure 5. PFS time of patients with MPLC and with IPM. The PFS time of patients with MPLC was increased compared with patients with IPM. However, the difference was not statistically significant (log-rank $\mathrm{P}=0.307$ ) PFS, progression-free survival; MPLC, multiple primary lung cancer; IPM, intrapulmonary metastasis.

(TKIs). For example, it was demonstrated that patients with EGFR/PIK3CA co-mutations had a less favorable PFS time during TKI therapy compared with patients with EGFR mutations alone $(33,35)$. Therefore, prognosis should be determined to analyze the implications of these alterations. The present study speculated that the genetic features of the IPM group may be associated with poor prognosis and TKI resistance. Despite genetic alterations requiring further confirmation, detecting genetic alterations may assist in the identification of MPLC or IPM and the subsequent application of therapy. No ALK gene alterations were detected in the present study, which is not congruent with previous results where concurrent EGFR mutations and ALK rearrangements in lung ADC were frequently observed (36). The lack of ALK rearrangements in the present study may have been characteristic of MLC, or perhaps the small cohort size affected the results. This was consistent with the results of Saab et al (1), who performed NGS and ALK FISH analyses on 52 lung adenocarcinomas tumors from 18 patients, none of the tumors harbored ALK gene rearrangements. In the present study, KRAS mutations were identified in 2 lesions of 2 patients and a NRAS mutation was detected in another patient. The rate $(2 / 17,11.76 \%)$ of KRAS detection was lower in the current study compared with that reported in Asian and Western populations (4-24 and $25 \%$, respectively) (37). The present results may therefore be associated with regional divergence and MLC characteristics.

$\mathrm{Hu}$ et al (20), demonstrated genomic evolution from atypical adenomatous hyperplasia to AIS, minimally invasive adenocarcinoma and ADC, and suggested that the neoplastic transformation of lung preneoplasia may be predominantly associated with the survival of best adapted subclones in a specific microenvironment. Therefore, in the present study, the differences and similarities of genetic alterations in patients with AIS and ADC were analyzed. The results of the non-parametric rank sum test revealed that the mean EGFR mutation abundance was higher in ADC group compared with the AIS group $(\mathrm{Z}=-2.845, \mathrm{P}=0.004)$. In addition, EGFR mutation location analysis in patients with AIS and ADC (EGFR non-L858R and L858R) demonstrated that the mutation detection rate of EGFR L858R in AIS was significantly higher compared with ADC. The mutation analysis of various tumor suppressor genes, including TP53, PIK3CA, RB1 and STK11, revealed a significant difference between the ADC group and the AIS group $\left(\chi^{2}=7.506, \mathrm{P}=0.006\right)$. Furthermore, a higher proportion of tumor suppressor gene mutations were observed in the ADC group compared with the AIS group. Concurrent EGFR and tumor suppressor gene mutations were significantly increased in the ADC group compared with the AIS group. Based on these results, the present study hypothesized that the mean EGFR mutation abundance, mutations of tumor suppressor genes and each mutation combined may serve an important role in the development of AIS into ADC. This may help elucidate the mechanisms underlying lung adenocarcinoma development and identify more effective targets for disease intervention.

The results of the present study revealed that the mean EGFR mutation abundance in ADC was significantly higher compared with AIS. This further indicates the importance of testing for genetic alterations. The current diagnosis of AIS and ADC is based on morphological assessment, which may not fully reflect the underlying pathology of these lesions. In future studies, more definitive endpoints, such as postsurgical recurrence and overall survival, should be integrated with molecular markers to improve the definition of indeterminate pulmonary nodule molecular subtypes and to assess their prognostic value (20). The present study recommends that patients with AIS should be genetically tested.

Previously, it was recommended that the proportion of tumor cells detected by NGS was $10 \%$ (17). The tumor cellularity of the majority of cases in the present study was $>10 \%$, and only five specimens of tumor cellularity were 6-10\%. EGFR, KRAS and TP53 mutations were also detected in patients in the present study. The results indicated that samples with $>6 \%$ tumor cellularity may be screened using NGS. However, there are various limitations to the present study. Firstly, although tumor cell content was assessed independently by two pathologists to obtain accurate data, the estimation of tumor cellularity can be subjective. Secondly, caution should be taken in analyzing tumor cellularity, particularly when small sample sizes are used. Thirdly, there were instances where tumor cellularity was $<10 \%$, and whether this affected detection was not determined. In addition, the majority of cases of MLC in the present study were lung ADC, and only two cases were SCC. This may be because ADC accounts for the vast majority of cases of lung cancer in China (38). Therefore, the results of the present study do not apply to patients with SCC. Future studies should be performed with a larger sample size to further support the conclusions of the present study and incorporate patients with SCC.

Intratumor heterogeneity is at least partially responsible for the discordance in mutation status between different sites of a tumor, and this heterogenicity may be a challenge for the application of targeted therapies (39). In studies by Li et al (17) and Normanno et al (18), HS values were used to assess intratumor mutational heterogeneity. The results of the current study indicated that intratumor heterogeneity existed in patients with MPLC and IPM. The mean HS value of EGFR in patients with IPM was higher compared with MPLC. Additionally, metastasis in patients with an EGFR HS $>1$ indicated that copy number variation may exist in the metastatic cells of IPM. No significant differences were identified in the TP53 HS value between the MPLC and IPM, with each 
exhibiting values $<1$, suggesting that mutations were present in a subpopulation of tumor cells. These results support the notion that EGFR mutations are more frequent in patients with IPM (17). Regression analysis revealed that a higher EGFR HS value and the male sex were associated with a greater risk of IPM, thus providing a potentially novel diagnostic marker to distinguish IPM from patients with MPLC.

In conclusion, the detection of genetic alterations using NGS may assist the identification of MPLC or IPM. Additionally, samples with $>6 \%$ tumor cellularity may be screened using NGS. Histological characteristics combined with genetic alterations may be an effective method to identify MPLC and IPM. MLC may have its own unique molecular characteristics, such as a higher rate and abundance of EGFR mutations, EGFR driver and tumor suppressor gene mutations combined, a lack of ALK mutations and a low rate RAS mutations, all of which may aid the identification of MPLC from IPM. The present study hypothesizes that mean EGFR mutation abundance, mutations of tumor suppressor genes and mutations of each combined may serve an important role in the development of AIS into ADC. This may help elucidate the mechanism of lung adenocarcinoma development to identify more effective measures of therapeutic intervention. Furthermore, the present study recommends that patients with AIS should be genetically tested.

\section{Acknowledgements}

Not applicable.

\section{Funding}

The present study was supported by The National Natural Science Foundation of China (grant no. 81602138) and The Beijing Hospitals Authority Youth Program (grant no. QML20180304).

\section{Availability of data and materials}

The datasets used and/or analyzed during the present study are available from the corresponding author upon reasonable request.

\section{Authors' contributions}

MJ and JL designed the current study. XC, JL, YW collected the data, MJ and JL were responsible of pathological study. YW, YG, YL and HZ performed the experiments preparation. YW followed up with the patients and prepared the figures and tables. XJ, YG, YL were responsible for the molecular study. $\mathrm{XC}, \mathrm{JL}$ and $\mathrm{XJ}$ analyzed and interpreted the data. XC analyzed the statistics and drafted the initial manuscript. XC, JL and XJ revised the manuscript. All authors gave final approval for publication. MJ took full responsibility for the work as a whole, including the study design, access to data, and the decision to submit and publish the manuscript.

\section{Ethics approval and consent to participate}

The present study was approved by the Medical Ethics Committee of Beijing Chaoyang Hospital, Capital Medical
University (Beijing, China; approval no. 2018-Scientific-311). A patient who came from Beijing signed the informed written consent as a representative. The remaining 16 patients were from other provinces of China, thus these patients were contacted via telephone to obtain verbal informed consent before participation in the present study.

\section{Patient consent for publication}

Not applicable.

\section{Competing interests}

The authors declare that they have no competing interests.

\section{References}

1. Saab J, Zia H, Mathew S, Kluk M, Narula N and Fernandes H: Utility of genomic analysis in differentiating synchronous and metachronous lung adenocarcinomas from primary adenocarcinomas with intrapulmonary metastasis. Transl Oncol 10: 442-449, 2017.

2. Nakata M, Sawada S, Yamashita M, Saeki H, Kurita A, Takashima S and Tanemoto K: Surgical treatments for multiple primary adenocarcinoma of the lung. Ann Thorac Surg 78: 1194-1199, 2004.

3. Pairolero PC, Williams DE, Bergstralh EJ, Piehler JM, Bernatz PE and Payne WS: Postsurgical stage I bronchogenic carcinoma: Morbid implications of recurrent disease. Ann Thorac Surg 38: 331-338, 1984.

4. Martini N, Bains MS, Burt ME, Zakowski MF, McCormack P, Rusch VW and Ginsberg RJ: Incidence of local recurrence and second primary tumors in resected stage I lung cancer. J Thorac Cardiovasc Surg 109: 120-129, 1995.

5. Aziz TM, Saad RA, Glasser J, Jilaihawi AN and Prakash D: The management of second primary lung cancers. A single centre experience in 15 years. Eur J Cardiothorac Surg 21: 527-533, 2002.

6. Schneider F and Dacic S: Histopathologic and molecular approach to staging of multiple lung nodules. Transl Lung Cancer Res 6: 540-549, 2017.

7. Detterbeck FC, Nicholson AG, Franklin WA, Marom EM, Travis WD, Girard N, Arenberg DA, Bolejack V, Donington JS, Mazzone PJ, et al: The IASLC lung cancer staging project: Summary of proposals for revisions of the classification of lung cancers with multiple pulmonary sites of involvement in the forthcoming eighth edition of the TNM classification. J Thorac Oncol 11: 639-650, 2016.

8. Amin MB, Edge SB, Greene FL, et al (eds): AJCC cancer staging manual. 8th edition. New York, Springer, 2017.

9. Donfrancesco E, Yvorel V, Casteillo F, Stachowicz ML, Patoir A, Tiffet O, Péoc'h M and Forest F: Histopathological and molecular study for synchronous lung adenocarcinoma staging. Virchows Arch 476: 835-842, 2020.

10. Patel SB, Kadi W, Walts AE, Marchevsky AM,Pao A, Aguiluz A, Mudalige T, Liu Z, Deng N and Lopategui J: Next-generation sequencing: A novel approach to distinguish multifocal primary lung adenocarcinomas from intrapulmonary metastases. J Mol Diagn 19: 870-880, 2017.

11. Schneider F, Derrick V, Davison JM, Strollo D, Incharoen P and Dacic S: Morphological and molecular approach to synchronous non-small cell lung carcinomas: Impact on staging. Mod Pathol 29: 735-742, 2016.

12. Li W, Qiu T, Ling Y, Gao S and Ying J: Subjecting appropriate lung adenocarcinoma samples to next-generation sequencing-based molecular testing: Challenges and possible solutions. Mol Oncol 12: 677-689, 2018.

13. Liu Y, Zhang J, Li L, Yin G, Zhang J, Zheng S, Cheung H, Wu N, Lu N, Mao X, et al: Genomic heterogeneity of multiple synchronous lung cancer. Nat Commun 7: 13200, 2016.

14. Xiao F, Zhang ZR, Wang XW, Liu DR, Guo YQ, Shi B, Song ZY and Liang CY: Applying comprehensive histologic assessment and genetic testing to synchronous multifocal lung adenocarcinomas and further survival analysis. Chin Med J (Engl) 132: 227-231, 2019. 
15. Murphy SJ, Aubry MC, Harris FR, Halling GC, Johnson SH, Terra S, Drucker TM, Asiedu MK, Kipp BR, Yi ES, et al: Identification of independent primary tumors and intrapulmonary metastases using DNA rearrangements in non-small-cell lung cancer. J Clin Oncol 32: 4050-4058, 2014.

16. Robinson JT, Thorvaldsdóttir H, Wenger AM, Zehir A and Mesirov JP: Variant review with the integrative genomics viewer. Cancer Res 77: e31-e34, 2017.

17. Li W, Qiu T, Guo L and Ying JM: Major challenges related to tumor biological characteristics in accurate mutation detection of colorectal cancer by next-generation sequencing. Cancer Lett 410: 92-99, 2017.

18. Normanno N, Rachiglio AM,Lambiase M,Martinelli E,Fenizia F, Esposito C, Roma C, Troiani T, Rizzi D, Tatangelo F, et al Heterogeneity of KRAS, NRAS, BRAF and PIK3CA mutations in metastatic colorectal cancer and potential effects on therapy in the CAPRI GOIM trial. Ann Oncol 26: 1710-1714, 2015.

19. Cheng H, Lei BF, Peng PJ, Lin YJ and Wang XJ: Histologic lung cancer subtype differentiates synchronous multiple primary lung adenocarcinomas from intrapulmonary metastases. J Surg Res 211: 215-222, 2017.

20. Hu X, Fujimoto J, Ying L, Fukuoka J, Ashizawa K, Sun W, Reuben A, Chow CW, McGranahan N, Chen R, et al: Multi-region exome sequencing reveals genomic evolution from preneoplasia to lung adenocarcinoma. Nat Commun 10: 2978, 2019.

21. Nik-Zainal S, Van Loo P, Wedge DC, Alexandrov LB, Greenman CD, Lau KW, Raine K, Jones D, Marshall J, Ramakrishna M, et al: The life history of 21 breast cancers. Cell 149: 994-1007, 2012

22. Shah SP, Morin RD, Khattra J, Prentice L, Pugh T, Burleigh A, Delaney A, Gelmon K, Guliany R, Senz J, et al: Mutational evolution in a lobular breast tumour profiled at single nucleotide resolution. Nature 461: 809-813, 2009.

23. GerlingerM,Rowan AJ,HorswellS, Math M,Larkin J,EndesfelderD, Gronroos E, Martinez P, Matthews N, Stewart A, et al: Intratumor heterogeneity and branched evolution revealed by multiregion sequencing. N Engl J Med 366: 883-892, 2012.

24. Anderson K, Lutz C, van Delft FW, Bateman CM, Guo Y, Colman SM, Kempski H, Moorman AV, Titley I, Swansbury J, et al: Genetic variegation of clonal architecture and propagating cells in leukaemia. Nature 469: 356-361, 2011.

25. Thunnissen E, Beasley MB, Borczuk AC, Brambilla E, Chirieac LR, Dacic S, Flieder D, Gazdar A, Geisinger K, Hasleton P, et al: Reproducibility of histopathological subtypes and invasion in pulmonary adenocarcinoma. An international interobserver study. Mod Pathol 25: 1574-1583, 2012.

26. Girard N, Deshpande C, Lau C, Finley D, Rusch V, Pao W and Travis WD: Comprehensive histologic assessment helps to differentiate multiple lung primary nonsmall cell carcinomas from metastases. Am J Surg Pathol 33: 1752-1764, 2009.

27. Girard N, Ostrovnaya I, Lau C, Park B, Ladanyi M, Finley D, Deshpande C, Rusch V, Orlow I, Travis WD, et al: Genomic and mutational profiling to assess clonal relationships between multiple non-small cell lung cancers. Clin Cancer Res 15: 5184-5190, 2009.

28. La Fleur L, Falk-Sörqvist E, Smeds P, Berglund A, Sundström M, Mattsson JS, Brandén E, Koyi H, Isaksson J, Brunnström H, et al: Mutation patterns in a population-based non-small cell lung cancer cohort and prognostic impact of concomitant mutations in KRAS and TP53 or STK11. Lung Cancer 130: 50-58, 2019.
29. Jakobsen JN, Santoni-Rugiu E, Grauslund M, Melchior L and Sørensen JB: Concomitant driver mutations in advanced EGFR-mutated non-small-cell lung cancer and their impact on erlotinib treatment. Oncotarget 40: 26195-26208, 2018.

30. Skov BG, Høgdall E, Clementsen P, Krasnik M, Larsen KR, Sørensen JB, Skov T and Mellemgaard A: The prevalence of EGFR mutations in non-small cell lung cancer in an unselected Caucasian population. APMIS 123: 108-115, 2015.

31. Shi Y, Li J, Zhang S, Wang M, Yang S, Li N, Wu G, Liu W, Liao G, Cai K, et al: Molecular epidemiology of EGFR mutations in asian patients with advanced non-small-cell lung cancer of adenocarcinoma histology-mainland China subset analysis of the PIONEER study. PLoS One 10: e0143515, 2015.

32. Shi Y, Au JS, Thongprasert S, Srinivasan S, Tsai CM, Khoa MT, Heeroma K, Itoh Y, Cornelio G and Yang PC: A Prospective, molecular epidemiology study of EGFR mutations in asian patients with advanced non-small-cell lung cancer of adenocarcinoma histology (PIONEER). J Thorac Oncol 9: 154-162, 2014.

33. Guibert N, Barlesi F, Descourt R, Lena H, Besse B, Beau-Faller M, Mosser J, Pichon E, Merlio JP, Ouafik L, et al: Characteristics and outcomes of patients with lung cancer harboring multiple molecular alterations: Results from the IFCT study biomarkers France. J Thorac Oncol 12: 963-973, 2017.

34. Tetsu O, Hangauer MJ, Phuchareon J, Eisele DW and McCormick F: Drug resistance to EGFR inhibitors in lung cancer. Chemotherapy 61: 223-235, 2016.

35. Eng J, Woo KM, Sima CS, Plodkowski A, Hellmann MD, Chaft JE, Kris MG, Arcila ME, Ladanyi M and Drilon A: Impact of concurrent PIK3CA mutations on response to EGFR tyrosine kinase inhibition in EGFR-Mutant lung cancers and on prognosis in oncogene-driven lung adenocarcinomas. J Thorac Oncol 10: 1713-1719, 2015.

36. Ulivi P, Chiadini E, Dazzi C, Dubini A, Costantini M, Medri L, Puccetti M, Capelli L, Calistri D, Verlicchi A, et al: Nonsquamous, non-small-cell lung cancer patients who carry a double mutation of EGFR, EML4-ALK or KRAS: Frequency, clinical-pathological characteristics, and response to therapy. Clin Lung Cancer 17: 384-390, 2016.

37. Riely GJ, Kris MG, Rosenbaum D, Marks J, Li A, Chitale DA, Nafa K, Riedel ER, Hsu M, Pao W, Miller VA and Ladanyi M: Frequency and distinctive spectrum of KRAS mutations in never smokers with lung adenocarcinoma. Clin Cancer Res 14: 5731-5734, 2008.

38. McIntyre A and Ganti AK: Lung cancer-A global perspective. J Surg Oncol 115: 550-554, 2017

39. Jiang L, Huang J, Morehouse C, Zhu W, Korolevich S, Sui D, Ge X, Lehmann K, Liu Z, Kiefer C, et al: Low frequency KRAS mutations in colorectal cancer patients and the presence of multiple mutations in oncogenic drivers in non-small cell lung cancer patients. Cancer Genet 206: 330-339, 2013.

This work is licensed under a Creative Commons Attribution-NonCommercial-NoDerivatives 4.0 International (CC BY-NC-ND 4.0) License. 